Wright State University

CORE Scholar

$5-1-2004$

\title{
Anomalous Capture and Emission from Internal Surfaces of Semiconductor Voids: Nanopores in SiC
}

David C. Look

Wright State University - Main Campus, david.look@wright.edu

Z-Q. Fang

S. Soloviev

T. S. Sudarshan

J. J. Boeckl

Follow this and additional works at: https://corescholar.libraries.wright.edu/physics

Part of the Physics Commons

\section{Repository Citation}

Look, D. C., Fang, Z., Soloviev, S., Sudarshan, T. S., \& Boeckl, J. J. (2004). Anomalous Capture and Emission from Internal Surfaces of Semiconductor Voids: Nanopores in SiC. Physical Review B, 69 (19), 195205.

https://corescholar.libraries.wright.edu/physics/207

This Article is brought to you for free and open access by the Physics at CORE Scholar. It has been accepted for inclusion in Physics Faculty Publications by an authorized administrator of CORE Scholar. For more information, please contact library-corescholar@wright.edu. 


\title{
Anomalous capture and emission from internal surfaces of semiconductor voids: Nanopores in $\mathrm{SiC}$
}

\author{
D. C. Look* and Z-Q. Fang \\ Semiconductor Research Center, Wright State University, Dayton, Ohio 45435, USA \\ S. Soloviev and T. S. Sudarshan \\ Department of Electrical Engineering, University of South Carolina, Columbia, South Carolina 29208, USA \\ J. J. Boeckl \\ Mater. and Mfg. Directorate, Air Force Research Laboratory, Wright-Patterson AFB, Ohio 45433, USA
}

(Received 9 March 2004; published 26 May 2004)

\begin{abstract}
Deep level transient spectroscopy in nanoporous, $n$-type $\mathrm{SiC}$ reveals a new type of deep $(\sim 0.8 \mathrm{eV})$ trap that can hold more than 100 electrons and that has anomalous capture and emission behavior. Here we quantitatively explain these effects with a new, general formalism that treats both emission and capture in the presence of dynamic energy barriers, resulting from the charging and discharging of states on the internal surfaces of voids, such as pores or nanopipes. The capture kinetics display a logarithmic time dependence over a certain filling range, as has often been observed in connection with dislocation-related trapping.
\end{abstract}

DOI: 10.1103/PhysRevB.69.195205

PACS number(s): 61.72.Qq, 71.55.Ht, 73.20.At, 73.50.Gr

Porous semiconductors, or semiconductors with voids, have long been in the forefront of research, because of their unique and often useful properties. The best example is, of course, porous $\mathrm{Si}^{1}{ }^{1}$ studied mainly for its light emission properties; however, other semiconductors, such as $\mathrm{SiC}$ and $\mathrm{GaN}$, are also now receiving much attention. For example, porous $\mathrm{SiC}$ (P-SiC), is presently being vigorously investigated as a buffer layer for epitaxial $\mathrm{SiC}$ and $\mathrm{GaN}$ growth, an active layer for gas sensors, and a cell-friendly transistor for in vivo applications. ${ }^{2-5}$ However, many of the electrical and optical properties are poorly understood, at present, partly because material control is very difficult. Recently, a correlation between carrier concentration and pore density in $n$-type $\mathrm{P}-\mathrm{SiC}$ has been observed, ${ }^{3,4}$ and this observation has been interpreted to mean that pores can act as electron traps. ${ }^{4}$ Traps in semiconductors can be conveniently studied by deep level transient spectroscopy (DLTS), ${ }^{6,7}$ and we have applied this technique to $\mathrm{P}-\mathrm{SiC}$. In comparison with the nonporous $\mathrm{SiC}$ (NP-SiC) case, most of the traps are the same and indeed are common to various $\mathrm{SiC}$ materials studied by other workers in the past. However, we also find a new trap of very high concentration $\left(\sim 6 \times 10^{17} \mathrm{~cm}^{-3}\right)$, appearing only in P-SiC. The DLTS signal from this new trap displays abnormally slow and nonexponential saturation with filling pulse length $\left(t_{p}\right)$, whereas most traps saturate rapidly and exponentially, as $\left[1-\exp \left(-e_{n} t_{p}\right)\right]$, where $e_{n}$ is the emission rate. (Note that most analysis software on commercial DLTS machines is based on exponential saturation and emission.) Besides the unusual saturation behavior, the DLTS spectrum moves to higher temperatures as $t_{p}$ increases, and also narrows with $t_{p}$. The standard DLTS modeling framework cannot explain these observances. It should be noted that similar effects have been observed in various semiconductors in which the traps lie along vertical threading dislocations, and thus form line charges. ${ }^{8,9}$ Indeed, some modeling of a logarithmic-type capture behavior has been carried out for dislocation-related traps, although we will show that such an analysis applies only over a restricted range of trap occupation.

In this work, we present a single, general formalism that describes both capture and emission behavior for traps that exist on the inner surfaces or interfaces of spherical or cylindrical structures embedded in semiconductor materials. Common structures of these shapes include pores, precipitates, and nanopipes. A very important case is cylindrical nanopipes (open-core screw dislocations) in GaN grown on $\mathrm{Al}_{2} \mathrm{O}_{3}$, which can sometimes generate states in the band gap, either due to dangling bonds or impurities that congregate nearby (the Cottrell atmosphere). ${ }^{10-12}$ Another widely studied example is spherical As precipitates in low-temperaturegrown molecular-beam epitaxial GaAs, which have been reported to have surface charge. ${ }^{13}$ A final example, the main subject of interest here, is nanopores in $\mathrm{SiC}$.

The porous $\mathrm{SiC}$ in this study was prepared by photoassisted electrochemical etching ${ }^{3}$ of $n$-type $6 \mathrm{H} \mathrm{SiC}$ obtained from Sterling Semiconductor, Inc. (now part of DowCorning Corp.). The electrolyte was a mixture of HF acid and ethanol. The resistivity of the starting material was about $0.2 \Omega$-cm, and the carrier concentration $\sim 10^{18} \mathrm{~cm}^{-3}$. The $\mathrm{C}-\mathrm{V}$ and DLTS data were obtained by means of a BioRad DL4600 DLTS apparatus, which operated over the temperature range $80-450 \mathrm{~K}$. From the $\mathrm{C}-\mathrm{V}$ data, the carrier concentration in the NP-SiC was uniform at about $10^{18} \mathrm{~cm}^{-3}$, whereas that in the P-SiC dropped to about $10^{17} \mathrm{~cm}^{-3}$ at a depth of about $80 \mathrm{~nm}$. Cross-sectional transmission electron microscopy (TEM), using a $200 \mathrm{kV}$ Phillips CM-200 instrument, was used to study the pore size and density. At depths of 50-100 $\mathrm{nm}$ below the surface, the pore radii ranged in size from 10-25 nm, with a density of about 5 $\times 10^{15} \mathrm{~cm}^{-3}$, increasing with depth. The sizes and densities of these pores are very typical of those found at the same depth (just below the so-called "skin layer") in other P-SiC samples. ${ }^{2-4}$ It should be noted that not all pores observed in 
$\mathrm{P}-\mathrm{SiC}$ are spherical, and in fact, several different shapes have been seen. ${ }^{5}$ However, we will consider only spherical and cylindrical pores here, because they are among the most common reported, and also because they have electrostatic potentials that are relatively easy to calculate.

We will show that a pore in $n$-type $\mathrm{SiC}$ can be viewed as a giant trap, due to deep acceptor states of sheet density $N_{\mathrm{SS}}$ on the inner surface of the pore. It will turn out that the value of $N_{\mathrm{SS}}$ necessary to fit our DLTS data is about 2.5 $\times 10^{12} \mathrm{~cm}^{-2}$, within the range of surface state densites reported for crystalline $\mathrm{SiC} .{ }^{14}$ The total number of traps per pore is then $4 \pi r_{p}^{2} N_{\mathrm{SS}}$, giving, in this case, about 125 total electrons for an average pore of radius $r_{p}=20 \mathrm{~nm}$. However, as more and more electrons are trapped, a negative (repulsive) potential $\phi_{\text {sph }}$ builds up, and the trapping rate diminishes. A spherical region depleted of free electrons, described by a local band bending of energy $\Phi_{\mathrm{sph}}=-e \phi_{\mathrm{sph}}\left(r_{p}\right)$, forms at the surface $\left(r=r_{p}\right)$ of the pore. The value of $\Phi$ can be calculated from Poisson's equation, which, for spherical pores, is most conveniently expressed in spherical coordinates. The symmetry obviates the need for angular terms, so that Poisson's equation becomes

$$
\frac{1}{r^{2}} \frac{d}{d r} r^{2} \frac{d \phi_{\mathrm{sph}}}{d r}=-\frac{\rho}{\varepsilon}=-\frac{e N_{D}}{\varepsilon},
$$

where $\rho$ is the charge density, $\varepsilon$ is the dielectric constant, and $N_{D}$ is the net donor density (actually, $N_{D}-N_{A}$, where $N_{A}$ is the acceptor concentration). We solve this equation in the depletion approximation, in which both $\phi$ and $d \phi / d r$ are required to vanish at $w$, defined as the radius of the total depleted region, ${ }^{6,7}$ including the pore radius. [In setting $\phi(w)=0$, we have arbitrarily set the zero of potential at the conduction-band edge in the neutral region. Thus, $\phi$ represents the "band bending."] Charge conservation requires that (4/3) $\pi\left(w^{3}-r_{p}^{3}\right) N_{D}=4 \pi r_{p}^{2} N_{\mathrm{SS}} f$, so that the final expression for energy, $\Phi=-e \phi$, can be shown to be

$$
\begin{aligned}
\Phi_{\mathrm{sph}}(r, f)= & \frac{e^{2} N_{\mathrm{SS}} r_{p} f}{\varepsilon}\left\{\frac{N_{D}}{r_{p} N_{\mathrm{SS}} f}\left[\frac{r^{2}-r_{p}^{2}}{6}\right]+\left[1+\frac{N_{D} r_{p}}{3 N_{\mathrm{SS}} f}\right]\right. \\
& \left.\times\left[\frac{r_{p}}{r}-1\right]+1+\frac{r_{p} N_{D}}{2 N_{\mathrm{SS}} f}\left[1-\left(1+\frac{3 N_{\mathrm{SS}} f}{r_{p} N_{D}}\right)^{2 / 3}\right]\right\},
\end{aligned}
$$

where $f$ is the fractional occupation of the trap states on the pore, i.e., $f=N_{\mathrm{SS}}^{-} / N_{\mathrm{SS}}$. Equation (2) holds for $r_{p} \leqslant r \leqslant w$, and is cast in a form which is convenient in that the first two terms drop out for $r=r_{p}$. (For $r \geqslant w, \Phi=0$.) In our case, $N_{D} \approx 10^{18} \mathrm{~cm}^{-3}$, so that $\Phi\left(r_{p}\right) \approx 0.2 \mathrm{eV}$, for $f=1$.

For cylindrical pores, the charge-conservation condition is $\pi\left(w^{2}-r_{p}^{2}\right) L N_{D}=2 \pi r_{p} L N_{\mathrm{SS}} f$, where $L$ is the pore length. By solving Poisson's equation in the cylindrical coordinate system [let $r^{2} \rightarrow r$, in Eq. (1)], we get, again for $r_{p} \leqslant r \leqslant w$,

$$
\begin{aligned}
\Phi_{\mathrm{cyl}}(r, f)= & \frac{e^{2} N_{\mathrm{SS}} r_{p} f}{2 \varepsilon}\left\{[ 1 + \frac { r _ { p } N _ { D } } { 2 N _ { \mathrm { SS } } f } ] \left[\ln \left(1+\frac{2 N_{\mathrm{SS}} f}{r_{p} N_{D}}\right)\right.\right. \\
& \left.\left.-2 \ln \left(\frac{r}{r_{p}}\right)\right]+\frac{\left(r^{2}-r_{p}^{2}\right) N_{D}}{2 r_{p} N_{\mathrm{SS}} f}-1\right\} .
\end{aligned}
$$

In this case, $\Phi\left(r_{p}\right) \approx 0.3 \mathrm{eV}$, for $f=1$.

The dynamic capture and emission processes can now be described by the usual master equation:

$$
\frac{d f}{d t}=-e_{n} f+c_{n}(1-f),
$$

where $e_{n}$ is the emission rate from filled traps, and $c_{n}$ is the capture rate to empty traps. Both rates will be affected (slowed down) by the band bending, as follows:

$$
\begin{gathered}
c_{n}(n, T, f)=\sigma v(T) n e^{-\Phi_{\mathrm{sph}}\left(r_{p}, f\right) / k T} \\
\equiv c_{n 0}(n, T) e^{-\Phi_{\mathrm{sph}}\left(r_{p}, f\right) / k T}, \\
e_{n}(T, f)=\sigma_{\mathrm{emis}} v(T) N_{\mathrm{CB}}(T) e^{-\left[E_{\mathrm{SS}}+\Phi_{\mathrm{sph}}\left(r_{p}, f\right)\right] / k T} \\
\equiv e_{n 0}(T) e^{-\Phi_{\mathrm{sph}}\left(r_{p}, f\right) / k T},
\end{gathered}
$$

where $v(T)=\left(8 k T / \pi m^{*}\right)^{1 / 2}$ is the thermal velocity, $N_{\mathrm{CB}}(T)=2\left(2 \pi m^{*} k T\right)^{3 / 2} / h^{3}$ is the effective conduction band density of states (in the Boltzmann approximation), $n$ is the free electron concentration, $\sigma$ is the capture cross section for a single trap, and $\sigma_{\text {emis }}=\left(g_{0} / g_{1}\right) \sigma \exp (\alpha / k)$, where $g_{0}$ and $g_{1}$ are the degeneracies of the unoccupied and occupied trap states, respectively, and $\alpha$ is a linear temperature coefficient: $E_{\mathrm{SS}}=E_{\mathrm{SS} 0}(1-\alpha T)$. The exponential term in Eq. (5) arises from the fact that the effective concentration of free electrons able to surmount the energy barrier at the surface of a pore is not $n$, but $n e^{-\Phi / k T}$. Equation (6) results from setting $d f / d t$ $=0$, in equilibrium, and then comparing the resulting equation, $f=1 /\left(1+e_{n} / c_{n}\right)$, with the relevant Fermi function.

From Eqs. (4)-(6), a general transcendental, integral equation, describing both capture and emission, can be written for $f(t)$ :

$$
\int_{f_{\alpha}}^{f_{\beta}} \frac{e^{\Phi_{\mathrm{sph}}\left(r_{p}, f\right) / k T}}{1-f\left[1+\frac{e_{n 0}(T)}{c_{n 0}(n, T)}\right]} d f=c_{n 0}(n, T)\left(t_{\beta}-t_{\alpha}\right) .
$$

In a DLTS experiment, the capture process is carried out by applying a forward bias to a normally reversed-biased Schottky barrier or $p-n$ junction. ${ }^{6,7}$ In reverse bias, the traps are in a region depleted of free electrons, and thus experience a very low free-electron concentration, $n=n_{r} \ll n_{b}$, where $n_{b}$ is the bulk (neutral) value, $10^{18} \mathrm{~cm}^{-3}$ in this case. Thus, $c_{n 0}\left(n_{r}, T\right)$ is very small, so that emission dominates and the traps are almost empty. Then, in forward bias, the traps are suddenly exposed to the bulk free-electron concentration $n$ $=n_{b}$ for a time $t_{p}$, the filling pulse length, and at the end of this pulse the filled fraction is defined as $f_{p}$. Thus, the trap filling process is described by solving Eq. (7) for $f_{p}$ under the conditions $f_{\alpha}=0, f_{\beta}=f_{p}, n=n_{b}, t_{\alpha}=0$, and $t_{\beta}=t_{p}$. [One convenient means of solving Eq. (7) is by use of the "root" function in Mathcad. ${ }^{15}$ ] 


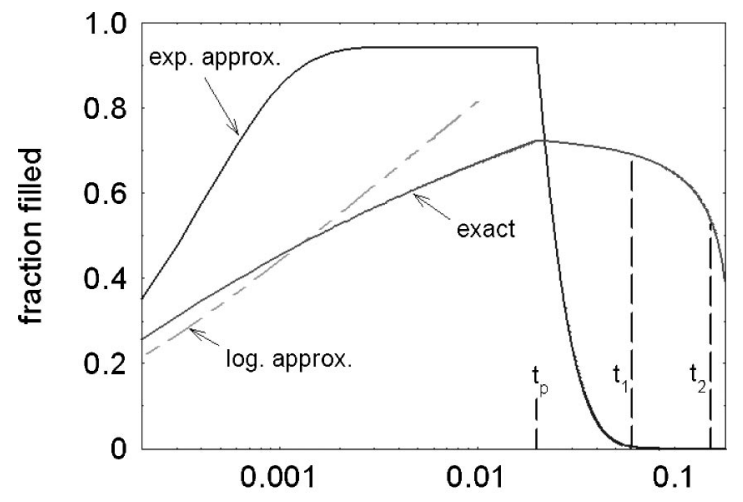

$t(s)$

FIG. 1. Fractional occupation, during capture and emission at $350 \mathrm{~K}$, of a pore in porous $\mathrm{SiC}$. An exact calculation is compared with exponential and logarithmic approximations. The filling pulse length is $t_{p}$, and the sampling points on the emission transient are $t_{1}$ and $t_{2}$, respectively.

When the filling pulse has ended at time $t_{p}$, i.e., by reapplying the reverse bias, the traps are once again suddenly exposed to a very small value of $n$, i.e., $n=n_{r}$. [Note that the solution of Eq. (7) is very insensitive to the exact value of $n_{r}$, as long as $n_{r} \ll n_{b}$.] The traps now emit their carriers, so that the original fractional occupation $f_{p}$ is now reduced to $f_{e}$, in total time $t_{p}+t_{e}$. Thus, in emission, Eq. (7) is solved for $f_{e}$ under the conditions $f_{\alpha}=f_{p}, f_{\beta}=f_{e}, n=n_{r}$, $t_{\alpha}=t_{p}$, and $t_{\beta}=t_{p}+t_{e}$. In the most common form of DLTS methodology, used in commercial instruments and often called the "boxcar" technique, ${ }^{6}$ the emission curve is evaluated at two points, $t_{1}$ and $t_{2}$, and the signal strength is measured as $S \equiv f\left(t_{1}\right)-f\left(t_{2}\right)$. Such a signal is simulated simply by solving Eq. (7) at two times, $t_{p}+t_{1}$ and $t_{p}+t_{2}$.

Before applying Eq. (7) to the problem at hand, it is instructive to solve it in two special cases, which apply to the majority of DLTS analyses, at the present time. In case I, the most common of all, we set $\Phi\left(r_{p}, f\right)=0$ (or a constant). Then, Eq. (7) immediately yields closed-form exponential capture and emission equations. The other special case of interest is realized under two conditions: (1) small $f$, such that the denominator of the integrand in Eq. (7) can be approximated by unity; and (2) $\Phi\left(r_{p}, f\right) \propto f$, which means that the large, bracketed terms in Eqs. 2 and 3, respectively, must be independent of $f$, i.e., a constant $K$. Then, Eq. (7) yields a logarithmic solution for $f$ [cf. Eq. (3) of Ref. 9], which has been seen experimentally for trapping along dislocation lines. ${ }^{8,9}$

We now return to the main problem at hand, i.e., pores in SiC. In Fig. 1, we compare the capture and emission solutions for three different cases: (1) an exact analysis [Eq. (7)]; (2) an exponential analysis [setting $\Phi_{\text {sph }}=0$, in Eq. (7)]; and (3) a logarithmic analysis $\left[\right.$ for $f \ll 1$, and $\Phi_{\text {sph }}$ $=K e^{2} N_{\mathrm{SS}} r_{p} f / \varepsilon$ in Eq. (7)]. To generate the curves, we have used some SiC parameters from the literature: $\mathrm{m}^{*} / \mathrm{m}_{0}$ $=0.4$, and $\varepsilon / \varepsilon_{0}=10$; some parameters measured by TEM or $C-V: \quad r_{p}=2 \times 10^{-6} \mathrm{~cm}, \quad N_{D} \approx n_{b} \approx 10^{18} \mathrm{~cm}^{-3}, \quad$ and $n_{e} \approx 10^{9} \mathrm{~cm}^{-3}$ (fit not sensitive to $n_{e}$ ); and some fitted parameters (i.e., those needed to fit the DLTS data of trap

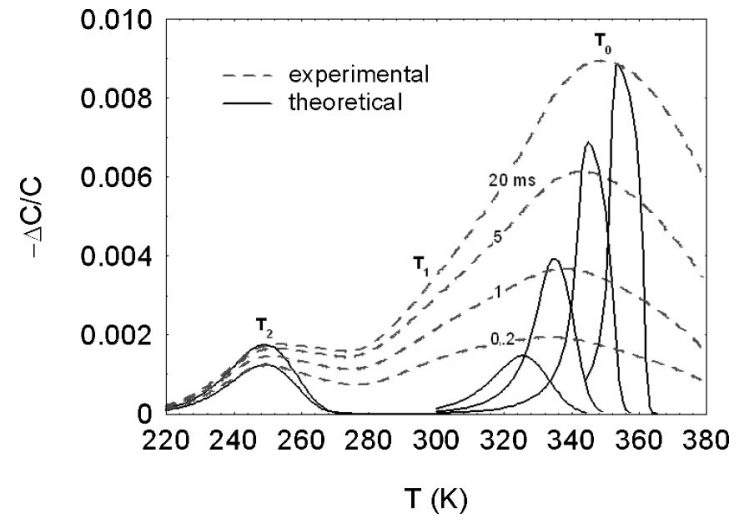

FIG. 2. Experimental (dashed lines) and theoretical (solid lines) DLTS curves for different filling-pulse lengths, 0.2, 2, 5, and $20 \mathrm{~ms}$, in porous SiC. Trap $T_{2}$ is a "normal" trap (impurity or point defect), which obeys exponential kinetics, and trap $T_{0}$ is related to the pores.

$T_{0}$ in Fig. 2): $N_{\mathrm{SS}}=2.5 \times 10^{12} \mathrm{~cm}^{-2}, E_{\mathrm{SS} 0}=0.8 \mathrm{eV}, \sigma=1$ $\times 10^{-22} \mathrm{~cm}^{2}$; and $\sigma_{\text {emis }}=3 \times 10^{-13} \mathrm{~cm}^{2}$. A filling pulse length $t_{p}=20 \mathrm{~ms}$ was assumed for the curves in Fig. 1. The exponential approximation is the one assumed in the vast majority of DLTS experiments, and, indeed, it works well for simple, isolated traps such as $T_{2}$ (cf. Fig. 2). However, it rises much too fast to explain the capture process of the pore-type traps ( $T_{0}$, in Fig. 2). The logarithmic approximation, on the other hand, works fairly well for filling fractions up to about 0.5 , but fails beyond that point. From the exact solution, it is seen that even at $t_{p}=20 \mathrm{~ms}$, complete saturation has not taken place. In emission, the exact solution is also much slower than the exponential solution, because at higher values of $f$ the emitting electrons experience a strong Coulomb barrier and are slowed down. In Fig. 1, we have also simulated a boxcar analysis on the BioRad DL4600 instrument by indicating a common set of sampling points, $t_{1}$ $=61.0 \mathrm{~ms}$ and $t_{2}=152.6 \mathrm{~ms}$, referenced with respect to $t_{p}$. This choice leads to an emission rate of $\ln \left(t_{2} / t_{1}\right) /\left(t_{2}-t_{1}\right)$ $=10 \mathrm{~s}^{-1}$ at the signal maximum of a trap such as $T_{2}$ (Fig. 2), which has an exponential emission. ${ }^{6,7}$ However, the emission for trap $T_{0}$ is far from exponential, so that the "standard" analysis will be highly inaccurate in this case.

The experimental DLTS data, for filling pulse lengths of 0.2, 1.0, 5.0, and $20 \mathrm{~ms}$, are shown as dashed lines in Fig. 2. Here we have plotted $\Delta C / C$, where $\Delta C=C\left(t_{1}\right)-C\left(t_{2}\right)$, and $C$ is the equilibrium capacitance under the reverse-bias condition, $V_{r}=-5 \mathrm{~V}$. It can be shown that $-\Delta C / C$ $\cong F_{\lambda} N_{T} / 2 N_{D}$, where $N_{T}$ is the trap concentration, and $F_{\lambda}$ is a factor which is close to unity for small trap concentrations $\left(N_{T} \ll N_{D}\right)$ and energies that are not too deep, but $<1$ otherwise. ${ }^{7}$ For our case, $N_{T}=4 \pi r_{p}^{2} N_{\mathrm{SS}} N_{p}$, where $N_{p}$ is the volume density of pores. From the TEM measurements, the sheet density of pores is about $3 \times 10^{10} \mathrm{~cm}^{-2}$, and the volume density $N_{p}$ is then, very approximately, $\left(3 \times 10^{10}\right)^{3 / 2}$ $\approx 5 \times 10^{15} \mathrm{~cm}^{-3}$. Thus, $N_{T} \approx 6 \times 10^{17} \mathrm{~cm}^{-3}$, and from this value and also $E_{T}=E_{\mathrm{SS}}=0.8 \mathrm{eV}$, we can calculate $F_{\lambda}=0.25{ }^{7}$ The actual DLTS signal is $\propto N_{T}\left[f\left(t_{1}\right)-f\left(t_{2}\right)\right]$, as shown in Fig. 1, and $f\left(t_{1}\right)-f\left(t_{2}\right)$ is calculated to be 0.646 at 
the peak of the 20-ms theoretical curve, in Fig. 2. Thus, from the TEM data, we would predict that $F_{\lambda} N_{T}\left[f\left(t_{1}\right)\right.$ $\left.-f\left(t_{2}\right)\right] / N_{D} \approx 0.10$, whereas we actually need a value of about 0.03 to fit the data at the peak, as shown. In other words, we need an $N_{T}$ value of about $2 \times 10^{17} \mathrm{~cm}^{-3}$ to fit the data, which is not outside the error of that determined by TEM $\left(6 \times 10^{17} \mathrm{~cm}^{-3}\right)$, considering that the latter value is a rather crude estimate.

The normalization factor for the 20-ms curve is now applied to the other three theoretical curves, and they reproduce their respective experimental peak magnitudes quite well. Furthermore, the temperature shifts are also well reproduced, giving strong validity to our model. Finally, both the experimental and theoretical curves become more narrow at larger values of $t_{p}$. The experimental curves are of course broader than their theoretical counterparts, because we have not considered the known variations in $r_{p}$, and the possible variations in $E_{\mathrm{SS}}$. The variation in $r_{p}$ is not likely the cause of the broadening, because neither a doubling nor a halving of the pore size moves the curves by more than a few K. On the other hand, an increase of $E_{\mathrm{SS}}$ from 0.80 to $0.85 \mathrm{eV}$ moves the curves up by almost $20 \mathrm{~K}$, which is sufficient to explain the line broadening. Indeed, a $\pm 0.05 \mathrm{eV}$ variation in $E_{\mathrm{SS}}$ seems quite reasonable, since some of the pores will undoubtedly be close enough to influence each other. It also should be noted that adding more traps at different values of $E_{\text {SS }}$ would also bring the total, fitted $N_{T}$ closer to the TEM estimate.

A useful parameter for porous materials is the porosity $P$, which for our sample can be immediately calculated as $P$
$=(4 / 3) \pi r_{p}^{3} N_{p} \approx 0.2$. It follows that about $20 \%$ of the carriers are depleted due to the loss of material in the voids; however, the total fractional volume depleted of free electrons is $(4 / 3) \pi w^{3} N_{p} \approx 0.8$, where $w\left(\approx 3.4 \times 10^{-6} \mathrm{~cm}\right)$ is the depletion radius, discussed earlier. This means that about $60 \%$ of the carriers are depleted due to the traps on the void surfaces, not the voids themselves. The predicted depletion is quite consistent with the $C-V$ measurements, which indicate that the average $n$ (averaged over the depleted regions ${ }^{3}$ ) has fallen from about $10^{18}$ to about $10^{17} \mathrm{~cm}^{-3}$ in the region sampled by the DLTS experiment.

In summary, we have developed a general formalism that quantitatively treats capture and emission processes in spherical and cylindrical semiconductor structures that can trap multiple charges on their surfaces. The trap filling and emission processes are then subject to a time-varying Coulomb barrier, which leads to nonexponential kinetics. The model shows that pores in $n$-type, porous $\mathrm{SiC}$ act as giant traps ( $>100$ electrons per pore) that strongly affect DLTS data and that can even render the material semi-insulating. A special case of the model predicts a logarithmic time dependence of capture, often applied in the past to dislocationrelated traps. Besides pores, other structures, such as nanopipes and precipitates, can be treated with our formalism.

\section{ACKNOWLEDGMENTS}

We wish to thank C. Wood for suggesting this problem, and B. Claflin, G. Farlow, and P. W. Yu for helpful discussions. D.C.L. and Z.Q.F. were supported by ONR DURINT Grant No. N00014-01-1-0715 (monitored by Dr. C. Wood).

*Electronic mail: david.look@wpafb.af.mil

${ }^{1}$ A. G. Cullis, L. T. Canham, and P. D. J. Calcott, J. Appl. Phys. 82, 909 (1997).

${ }^{2}$ A. Sagar, C. D. Lee, R. M. Feenstra, C. K. Inoki, and T. S. Kuan, J. Appl. Phys. 92, 4070 (2002).

${ }^{3}$ S. Soloviev, T. Das, and T. S. Sudarshan, Electrochem. SolidState Lett. 6, G22 (2003).

${ }^{4}$ P. A. Ivanov, M. Mynbaeva, and S. E. Saddow, Semicond. Sci. Technol. 19, 319 (2003).

${ }^{5}$ Y. Shishkin, W. J. Choyke, and R. P. Devaty, Mater. Sci. Forum 457, 1467 (2004).

${ }^{6}$ D. V. Lang, J. Appl. Phys. 45, 3023 (1974).

${ }^{7}$ D. C. Look and J. R. Sizelove, J. Appl. Phys. 78, 2848 (1995).

${ }^{8}$ T. Wosinski, J. Appl. Phys. 65, 1566 (1989).

${ }^{9}$ A. Hierro, A. R. Arehart, B. Heying, M. Hansen, J. S. Speck, U.

K. Mishra, S. P. DenBaars, and S. A. Ringel, Phys. Status Solidi B 228, 309 (2001).

${ }^{10}$ J. Elsner, R. Jones, P. K. Sitch, V. D. Borezag, M. Elstner, Th. Frauenheim, M. I. Heggie, S. Öberg, and P. R. Briddon, Phys. Rev. Lett. 79, 3672 (1997).

${ }^{11}$ Z. Liliental-Weber, Y. Chen, S. Ruvimov, and J. Washburn, Phys. Rev. Lett. 79, 2835 (1997).

${ }^{12}$ I. Arslan and N. D. Browning, Phys. Rev. Lett. 91, 165501 (2003).

${ }^{13}$ A. C. Warren, J. M. Woodall, J. L. Freeouf, D. Grischkowsky, D. M. McInturff, M. R. Melloch, and N. Otsuka, Appl. Phys. Lett. 57, 1331 (1990).

${ }^{14}$ P. A. Ivanov, M. E. Levinshtein, J. W. Palmour, and S. L. Rumyantsev, Semicond. Sci. Technol. 15, 164 (2000).

${ }^{15}$ Mathsoft, 101 W. Main St., Cambridge, MA 02142. 